\title{
OPEN Retinal hyperspectral imaging in the 5xFAD mouse model of Alzheimer's disease
}

\author{
Jeremiah K. H. Lim ${ }^{1,4}$, Qiao-Xin Li², Tim Ryan ${ }^{3}$, Phillip Bedggood ${ }^{1}$, Andrew Metha ${ }^{1}$, \\ Algis J. Vingrys ${ }^{1}{ }^{1}$, Bang V. Bui ${ }^{1}$ \& Christine T. O. Nguyen ${ }^{1 \times 1}$
}

Hyperspectral imaging of the retina has recently been posited as a potentially useful form of spectroscopy of amyloid-beta (A $\beta$ ) protein in the eyes of those with Alzheimer's disease (AD). The concept of using the retina as a biomarker for $A D$ is an attractive one, as current screening tools for $A D$ are either expensive or inaccessible. Recent studies have investigated hyperspectral imaging in $A \beta$ models however these studies have been in younger mice. Here we characterised hyperspectral reflectance profile in 6 to 17 months old 5xFAD mice and compare this to $A \beta$ in isolated preparations. Hyperspectral imaging was conducted across two preparations of $A \beta$ using a custom built bench ophthalmoscope. In the in vitro condition, $1 \mathrm{mg}$ of purified human $A \beta 42$ was solubilised and left to aggregate for $72 \mathrm{~h}$. This soluble/insoluble $A \beta$ mixture was then imaged by suspending the solution at a pipette tip and compared against phosphate buffered saline (PBS) control ( $n=10$ ROIs / group). In the in vivo condition, a 5xFAD transgenic mouse model was used and retinae were imaged at the age of $6(n=9), 12(n=9)$ and 17 months $(n=8)$ with age matched wildtype littermates as control $(n=12$, $n=13, n=15$ respectively). In the vitro condition, hyperspectral imaging of the solution showed greater reflectance compared with vehicle $(p<0.01)$, with the greatest differences occurring in the short visible spectrum $(<500 \mathrm{~nm})$. In the in vivo preparation, $5 x F A D$ showed greater hyperspectral reflectance at all ages $(6,12,17$ months, $p<0.01)$. These differences were noted most in the short wavelengths at younger ages, with an additional peak appearing at longer wavelengths $(\sim 550 \mathrm{~nm})$ with advancing age. This study shows that the presence of $A \beta$ (soluble/insoluble mixture) can increase the hyperspectral reflectance profile in vitro as well as in vivo. Differences were evident in the short wavelength spectrum $(<500 \mathrm{~nm}$ ) in vitro and were preserved when imaged through the ocular media in the in vivo conditions. With advancing age a second hump around $\sim 50 \mathrm{~nm}$ became more apparent. Hyperspectral imaging of the retina does not require the use of contrast agents and is a potentially useful and non-invasive biomarker for AD.

Current biomarkers of Alzheimer's disease (AD) are either invasive, expensive or have limited accessibility outside of research. This has led to searches for more accessible and lower-cost alternatives with recent advances in plasma biomarkers ${ }^{1,2}$ as well as the search for pathophysiological biomarkers in other locations such as the eye $^{3,4}$. Although the presence of amyloid-beta peptide $(\mathrm{A} \beta)$ has previously been reported in the retinae of human post mortem eyes that were free of ocular disease, it was not confirmed if these donors indeed had $\mathrm{AD}^{5}$. Using curcumin as an amyloid binding agent for histology, retinal $A \beta$ was detected in the post-mortem retinae of $A D$ patients $^{6,7}$. Co-labelling with $A \beta$-specific antibodies (11A5-B10, 12F4, 4G8, 6E10) and thioflavin-S support the presence of this hallmark in the retina ${ }^{6,8}$. However, other investigators using routine immunohistochemistry methods (primary antibody, $4 \mathrm{G} 8$ and $6 \mathrm{~F} / 3 \mathrm{D}$ ) were unable to detect $\mathrm{A} \beta$ in the retinae of patients with confirmed $\mathrm{AD}^{9-11}$. Preclinical studies, in a range of mouse models genetically engineered to express human $\mathrm{A} \beta$ in the brain, find that $A \beta$ also accumulates in the retinae ${ }^{12-22}$.

Given the vicissitudes in detecting $A \beta$ in human retina, Koronyo et al. ${ }^{6}$ explored the possibility of in vivo fluorescence imaging of $A \beta$ using curcumin as a contrast agent to bind insoluble and soluble fractions of $A \beta$, and were successful in demonstrating a scattered distribution of labelled deposits in living human eyes using

${ }^{1}$ Department of Optometry and Vision Sciences, University of Melbourne, Parkville, VIC 3010, Australia. ${ }^{2}$ Florey Institute of Neuroscience and Mental Health, Parkville, VIC 3010, Australia. ${ }^{3}$ SAXS/WAXS Beamline, ANSTO/Australian Synchrotron, Clayton 3168, Australia. ${ }^{4}$ Optometry and Vision Science, College of Nursing and Health Sciences, Flinders University, Bedford Park, SA 5042, Australia. ${ }^{\boxplus}$ email: christine.nguyen@ unimelb.edu.au 
scanning laser ophthalmoscopy. These investigators also used transmission electron microscopy to confirm that $\mathrm{A} \beta$ assembled into elongated fibrillar structures, reflecting depositions seen in the brain. Such results signal an encouraging step toward clinical utility; however, the need for a contrast agent reduces the practicality of retinal imaging as a screening tool, particularly with regards to cost, time and potential side-effects.

To date, there have been several attempts to exploit the fibril-associated molecular deposition of A $\beta$ to permit detection without the use of extraneous contrast. In post-mortem eyes, Campbell and co-workers distinguished $\mathrm{AD}$ retinae from controls in a canine model of $\mathrm{AD}$ using non-invasive imaging of retinal birefringence arising from fibrillar $\mathrm{A} \beta$ deposition with the aid of cross polarisers ${ }^{23-25}$.

The scattering of light by accumulations of small, structured molecules in biological tissues can also manifest useful wavelength-dependencies ${ }^{26,27}$. In this regard, hyperspectral imaging (HSI) employs the principles akin to standard spectroscopy, which is used to determine the constituents of a sample or material by analysing absorption of specific narrow band wavelengths. Initially developed in the late 1960's for remote sensing; HSI has gained traction in other fields ranging from biomedical imaging to archaeology. More and Vince ${ }^{27}$ suggested a scheme to exploit HSI for amyloid detection in AD. Using a hyperspectral darkfield microscope with a broadband light source to image an ex vivo preparation of SH-SY5Y human neuroblastic cells, they showed that the addition of soluble $A \beta 42(250 \mathrm{nM})$ produced a reduction in the spectra for wavelengths between 450 and $530 \mathrm{~nm}$. Applying this technique to ex vivo brain slices from human $\mathrm{AD}$ and ex vivo retinae from young APP/PS1 mice (without brain plaques), the authors showed a reduction in spectral reflectance at shorter wavelengths $(<580 \mathrm{~nm}) \mathrm{com}$ pared with control tissue ${ }^{27}$. These changes were attributed to the presence of soluble amyloid, small particles which are known to scatter light in tissue in a wavelength dependent fashion ${ }^{26}$.

In a follow-up to their ex vivo study, the authors modified and applied their approach for in vivo imaging of APP/PS1 transgenic AD mouse retinae ${ }^{28}$. Using a "topical endoscopic fundal imaging (TEFI) system" they identified decreased reflectance at short wavelengths $(<570 \mathrm{~nm})^{28}$ in 7 month old APP/PS1 mice. They attributed these reflectance changes to increased soluble $A \beta$, as insoluble $A \beta$ deposits are only sparsely found in retina and in older (9-11 months) APP/PS1 mice ${ }^{7,29}$.

More recently, Hadoux et al. ${ }^{30}$ compared hyperspectral signatures in 15 people with mild cognitive impairment (MCI) and high A $\beta$ burden quantified using positron emission tomography (PET) with 20 age-matched controls. In this cross-sectional study, using modelling to account for individual variation in factors such as macular pigmentation and retinal oxygenation, they were able to detect a difference in the spectral profile between the MCI and control group. Hadoux et al. ${ }^{30}$ also found a similar difference in retinal reflectance between 9 to 14 month old controls and 5xFAD mice, a model of $\mathrm{AD}^{31}$.

The data of More et al. ${ }^{28}$ show that APP/PS1 mice retinae indicate progressively more scatter with age (3 to 8 -month-old), which they suggest is consistent with increasing soluble amyloid content. Mice used in More et al. ${ }^{28}$ were relatively young, and as such whether similar differences are still detectable at much older ages is not known. How advancing age-related factors such as optical media and retinal changes impact hyperspectral reflectance profile has yet to examined. By examining 5xFAD mice, we hope to show that HSI reflectance profiles provide useful information in a widely used murine model of amyloid deposition. Here, we examine age-related change to the HSI reflectance profile in 6,12 and 17 months of age wildtype and 5xFAD mice which approximate preclinical, moderate and severe $\mathrm{AD}$ stages ${ }^{32-34}$ and exhibit retinal $\mathrm{A} \beta$ staining ${ }^{22}$.

\section{Methods}

Two preparations of $A \beta$ underwent hyperspectral imaging on the same platform. Firstly, an in vitro preparation of purified human $\mathrm{A} \beta 42$, and secondly an in vivo preparation in a transgenic $5 \mathrm{xFAD}$ mouse model. Both preparations and all ages ${ }^{22}$ contained a mixture of soluble and insoluble $A \beta$. All experimental procedures abided by the "Australian code of practice for the care and use of animals for scientific purposes" as set out by the National Health and Medical Research Council of Australia (2013). Ethics approval was obtained from the Howard Florey Institute Animal Ethics Committee (Approval number: 13-068-UM). In addition, permission was sought from the Office for Research Ethics and Integrity for a notifiable low risk dealing approval (IBC reference: 2013/050) for the use of genetically modified organisms in accordance with the Australian Gene Technology Act (2000) and Gene Technology Regulations (2001). This study was carried out in compliance with the ARRIVE guidelines ${ }^{35}$. Experimenters were blinded to the treatment groups during data collection and analysis. 5xFAD and wild type littermates were randomised within blocks for time and day of experimentation.

In vitro preparation of $\mathbf{A} \beta \mathbf{4 2}$. Powdered purified human $\mathrm{A} \beta 42$ (Sigma A9810, St Louis, MO, USA) weighing $1 \mathrm{mg}$ was suspended in $100 \mu \mathrm{l}$ of $1 \mathrm{M}$ of sodium hydroxide $(\mathrm{NaOH})$ and incubated for $3 \mathrm{~min}$ at room temperature. The solution was diluted in $350 \mu \mathrm{l}$ of $\mathrm{dH}_{2} \mathrm{O}$ and sonicated in a water bath at room temperature for $5 \mathrm{~min}$. For neutralisation, $50 \mu \mathrm{l}$ of $10 \times$ phosphate buffered saline (PBS, pH 7) was added. The solution was then centrifuged (Centrifuge 5415D, Eppendorf AG, Hamburg, HH, Germany) at $14,000 \times g$ for $10 \mathrm{~min}$. The supernatant $(450 \mu \mathrm{l})$ was removed to allow for the exclusion of undissolved particulate matter. The optical density of the supernatant was measured at $214 \mathrm{~nm}$ using a spectrophotometer (UV-VIS 2700, Shimadzu, Chiyoda-ku, Tokyo, Japan). This was used to determine the concentration using the Beer-Lambert law (Eq. 1).

$$
A=\varepsilon . c . l
$$

where $\mathrm{A}=$ absorbance, $\varepsilon=$ extinction coefficient $\left(\mathrm{M}^{-1} \cdot \mathrm{cm}^{-1}\right), c=$ concentration $(\mathrm{M})$ and $l=$ path length $(\mathrm{cm})$. Using an extinction coefficient for $A \beta 1-42$ of $95,452 \mathrm{M} / \mathrm{cm}^{36}$ and a known path length of $1 \mathrm{~cm}$, we were able to determine the concentration of the solution, which was typically $70-80 \%$ of the peptide.

Using a combination of amyloid-binding dye (Thioflavin-T), size exclusion chromatography and transmission electron microscopy, Ryan and colleagues showed that soluble $\mathrm{A} \beta$ prepared this way aggregated after $20-\mathrm{h}^{37,38}$. 
To ensure that the solution contained a mixture of aggregated components, the $\mathrm{A} \beta$ solution was allowed to rest at $4{ }^{\circ} \mathrm{C}$ for a minimum of $72 \mathrm{~h}$ prior to measurement. This produced a solution that contained both soluble and insoluble $\mathrm{A} \beta$.

In vivo $A \beta$ assessment in 5xFAD mice. The Tg(APPSwFlLon,PSEN1*M146L ${ }^{\star}$ L286V)6799Vas/Mmjax) mouse model of $\mathrm{AD}$, also known as " $5 \mathrm{xFAD}$ " was used in this study due to its well characterised phenotype " $^{31}$. These mice were bred on a congenic C57BL/6 J background and do not contain the Pde6brd1 retinal degeneration allele. This model has 5 familial human AD genes and express human APP and A $\beta$ at an early age, allowing for the full-time course of AD development to be studied in a relatively short timeframe. A subset of the animals assessed in this study have been shown to exhibit soluble and insoluble $A \beta$ in the retina and brain ${ }^{22}$ which is in agreement with the literature ${ }^{14,30,39}$. Retinal hyperspectral imaging was conducted at 3 ages, namely $6(5 x F A D$, $\mathrm{n}=9$; WT $\mathrm{n}=12), 12(5 \mathrm{xFAD}, \mathrm{n}=9$; WT $\mathrm{n}=13)$ and 17 months of age $(5 \mathrm{xFAD}, \mathrm{n}=8$; WT $\mathrm{n}=15)$. These timepoints were chosen as behavioural studies in $5 \mathrm{xFAD}$ mice have indicated that they approximate preclinical, moderate and severe $\mathrm{AD}$, respectively ${ }^{32-34}$. The sample sizes are in agreement with previous power calculations and hyperspectral analysis from 5xFAD mice which spanned the middle age range (9 to 14 months old) of this group $^{30}$. Mice were group housed in well-ventilated conditions where the ambient temperature was maintained at $20^{\circ} \mathrm{C}$. A $12 \mathrm{~h} \mathrm{light/dark} \mathrm{cycle} \mathrm{was} \mathrm{used.} \mathrm{Food} \mathrm{(Baraston} \mathrm{mouse} \mathrm{pellets,} \mathrm{Ridley} \mathrm{Corporation,} \mathrm{Melbourne,} \mathrm{VIC,}$ Australia) and water were provided ad libitum. Ambient light levels were maintained at a low average level of $50 \mathrm{~lx}$ to prevent retinal damage ${ }^{40}$ and minimise stress ${ }^{41}$.

Animal handling and anaesthesia: Prior to procedures mice were anaesthetised using ketamine : xylazine anaesthesia (60:5 mg/kg, i.p. Therapon, Burwood, VIC, Australia). Topical anaesthetic and mydriatic eyedrops (Alcaine $0.5 \%$, Mydriacyl 0.5\% respectively, Alcon Laboratories, Frenchs Forest, NSW, Australia) were instilled. Animals were then placed onto a heated platform to maintain body temperature. All imaging was completed within 20 min of anaesthesia in order to maximise anterior eye clarity. During imaging, eyes were kept moist using Genteal gel (Alcon Laboratories, Frenchs Forest, NSW, Australia) and a coverslip, which prevents the development of cataracts and corneal dehydration.

Hyperspectral imaging platform. The custom-built bench ophthalmoscope uses a $150 \mathrm{~W}$ xenon light source with a fast switching monochromator (Polychrome V, Till Photonics, Hillsboro, OR, USA) as a hyperspectral light source (Supplementary Fig. 1). The light source has an intensity profile which varied by wavelength (Supplementary Fig. 2). The light source was placed perpendicular to the front of the eye or the in vitro preparation thereby providing bright-field flood illumination of the specimen imaged. A semi-reflective pellicle beam splitter (BP245B1, ThorLabs, Newton, NJ, USA) directs $45 \%$ of the incident light into the rodent eye. Light from the retina is returned through diffuse reflection and passes through the pellicle again. A condensing lens focuses the image directly onto a scientific complementary metal oxide semiconductor (CMOS) chip (Andor Technology, Belfast, UK). The light source and monochromator is capable of supplying visible light for retinal imaging using $10 \mathrm{~nm}$ bandwidths and is able to sweep out $1 \mathrm{~nm}$ steps centred from 320 to $680 \mathrm{~nm}$. Settling time for each step was $100 \mathrm{~ms}$, so that a full spectral series of 16-bit monochromatic images at each of 361 wavebands were acquired in $36.1 \mathrm{~s}$.

Hyperspectral images collected by the bench ophthalmoscope are influenced by the light output from the Polychrome V monochromator as well as the pellicle reflectance (light input arm) and transmittance (imaging arm). To correct for changes accrued in the imaging arm we divided by the pellicle transmittance profile as specified by the manufacturer; to correct for changes accrued in light input we used a power meter to measure irradiance in the plane of the pupil (therefore accounting for both the pellicle's reflectance to the eye and spectral variations in the polychrome light source). All raw in vivo and in vitro spectra were divided by these factors (Eq. 2). See Supplementary Fig. 2 for further details.

$$
\operatorname{Correctedintensity}(\lambda)=\frac{\text { Rawintensity }(\lambda)}{\text { Pellicetransmittance }(\lambda) * \operatorname{Lightinput}(\lambda)}
$$

To establish the safety of the device on the retina, a digital photometer (Model IL1700, International Light Technologies Inc., Newburyport, MA, USA) was used to compute the power density at the brightest wavelength $(475 \mathrm{~nm})$, which produced an output of $708.8 \mu \mathrm{W} / \mathrm{cm}^{2}$. The maximum pupil diameter measured was $2.3 \mathrm{~mm}$, which approximates $30 \mu \mathrm{W}$ of light reaching the retina. This level is considered safe based on the international electrotechnical commission 60,825 values for maximum permissible exposure ${ }^{42}$.

Image processing. Images were analysed using open source image analysis software, FIJI ${ }^{43}$. In vitro and in vivo images were exported as 16-bit greyscale Tag Image File Format (TIFF) stacks comprised of 361 images. Stacks were registered (using StackReg: Translation) to ensure that the same region of interest (ROI) was analysed in each image.

For in vitro $A \beta$ samples, square $(10 \times 10$ pixels) ROIs including and excluding visible amyloid aggregates were analysed for spectral intensity (Fig. 1a). For in vivo analysis, whole retinal images were manually masked to remove the major blood vessels and the optic nerve. The remaining image which represents retinal tissue and small blood vessels was analysed for hyperspectral reflectance. For quantification of in vitro and in vivo samples, hyperspectral profiles were first baseline corrected by subtracting the baseline light intensity at $380 \mathrm{~nm}\left(\lambda_{\text {base }}\right)$, and then normalised to the reference wavelength $\left(\lambda_{\text {ref }}=670 \mathrm{~nm}\right) .670 \mathrm{~nm}$ was chosen as our in vitro analysis showed that $\mathrm{A} \beta$ had a similar light intensity to vehicle at this wavelength (Fig. 1). This wavelength has also 

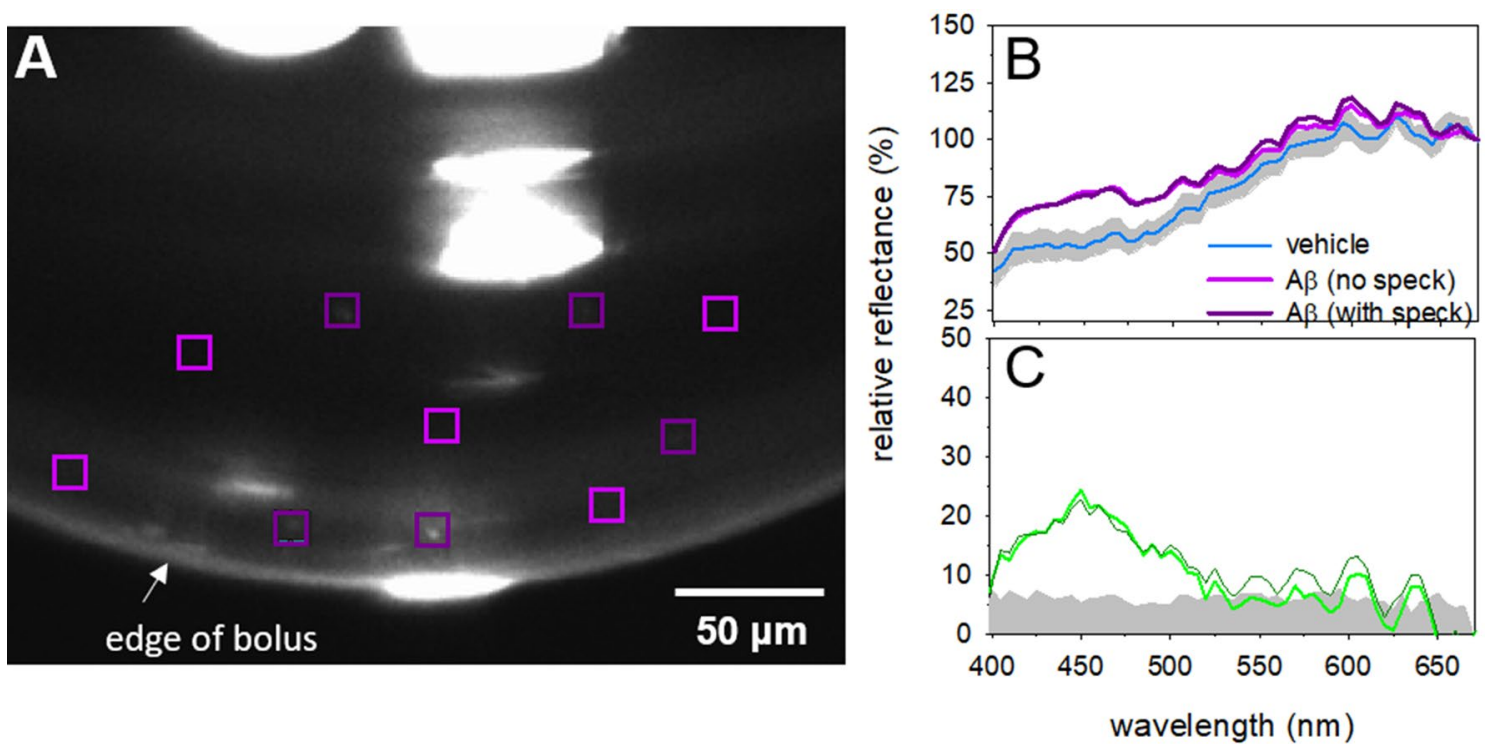

Figure 1. Spectral profile of isolated human A $\beta 42$. a A representative image of a $10 \mu$ droplet of $250 \mu \mathrm{M}$ $\mathrm{A} \beta$ solution. Regions of interest $(10 \times 10$ pixels, coloured squares) were chosen to either include visible $A \beta$ aggregations (white specks, dark purple) or avoid visible A $\beta$ aggregations (light purple). b Hyperspectral profiles of in vitro $A \beta$ preparation either including visible aggregations (dark purple, $n=10$ ROIs) or excluding visible aggregations (light purple, $\mathrm{n}=10 \mathrm{ROIs}$ ) and PBS vehicle control (blue line with grey shaded region representing, mean $\pm 95 \% \mathrm{CL}, \mathrm{n}=10 \mathrm{ROIs})$. $\mathrm{c}$ The difference between the $\mathrm{A} \beta$ preparation and control are represented in the average residual plot (dark green includes visible aggregates, light green excludes visible aggregates) with the grey area representing 95\% CI around the vehicle group only to aid visualisation.

previously been shown to be less sensitive to scatter in biological tissues ${ }^{28}$, though this may also be the case for longer wavelengths ${ }^{27}$.

To topographically visualise spatial distribution of hyperspectral change across the retina in 5xFAD mice, masked images were baseline corrected by subtracting the baseline image $\left(\lambda_{\text {base }}=380 \mathrm{~nm}\right)$ from the image stack. Processed images were analysed for $\mathrm{A} \beta\left(\lambda_{A \beta}=475 \mathrm{~nm}\right.$ as determined from the in vitro and in vivo $\mathrm{A} \beta$ imaging, Fig. 1b, 4a respectively) and expressed relative to the reference wavelength $\left(\lambda_{\text {ref }}\right.$ at $670 \mathrm{~nm}$; Eq. 3).

$$
\operatorname{Spectral} \Delta=\frac{\left(\lambda_{\mathrm{A} \beta}-\lambda_{\text {base }}\right)}{\left(\lambda_{\text {ref }}-\lambda_{\text {base }}\right)}
$$

The resulting ratio plots (Fig. 3) are colour coded to produce a topographical heat map (MATLAB R2013a, MathWorks, Natick, MA, USA) in order to highlight regions containing maximal differences in the spectra between $5 x F A D$ and WT.

\section{Statistical analysis}

Normality of the data was established using a Kolmogorov-Smirnov test. A Grubbs test was used to identify outliers. Statistical comparisons (Supplementary Table $1 \& 2$ ) were carried out using repeated measures two-way ANOVA to establish differences between combinations of $A \beta$ model and wavelength. Geisser-Greenhouse correction was applied as sphericity was not assumed. Post-hoc analysis was corrected for multiple comparisons by controlling the false discovery rate using a two stage linear step up procedure appropriate for overall recommendations based on multiple inferences as per Benjamini et al ${ }^{44,45} \mathrm{An}$ alpha of 0.05 was considered to be statistically significant. Statistical analyses were carried out in Prism 6 (GraphPad, San Diego, CA, USA).

\section{Results}

Spectral profile of in vitro $\mathbf{A} \beta \mathbf{4 2}$. The first aim was to determine if isolated human $A \beta 42$, containing a mixture of soluble and insoluble components, had a HSI profile $(380-680 \mathrm{~nm})$ distinguishable from vehicle. Two $10 \mu \mathrm{l}$ boli of A $\beta 42(2 \mathrm{mg} / \mathrm{ml})$ and PBS vehicle were suspended from the tip of a pipette. Figure 1a illustrates that visible aggregates (dark purple ROIs) can be seen in the $A \beta$ solution. These are approximately $\sim 1-2 \mu \mathrm{m}$ in size which would represent a large aggregate. The surrounding darker areas may contain smaller insoluble and soluble aggregates below the magnification resolution of the system. As such to ensure both soluble and insoluble $A \beta$ was captured, 5 ROIs $(10 \times 10$ pixel size) were selected that contained a visible $A \beta$ aggregate (insoluble $A \beta$ with the surrounding background region including soluble $A \beta$ ) and 5 ROIs selected that did not contain a visible $A \beta$ aggregate (soluble predominant, no visible insoluble specks seen) were selected from each $A \beta$ drop. Note that it is likely that both types of ROIs ("with speck" and "no speck") contain a mixture of soluble and insoluble A $\beta$, but the "with speck" ROIs include a higher proportion of insoluble given the larger visible aggregate. Phosphate buffered saline (PBS) was measured as vehicle control, with 5 ROIs in each of two droplets assessed. 


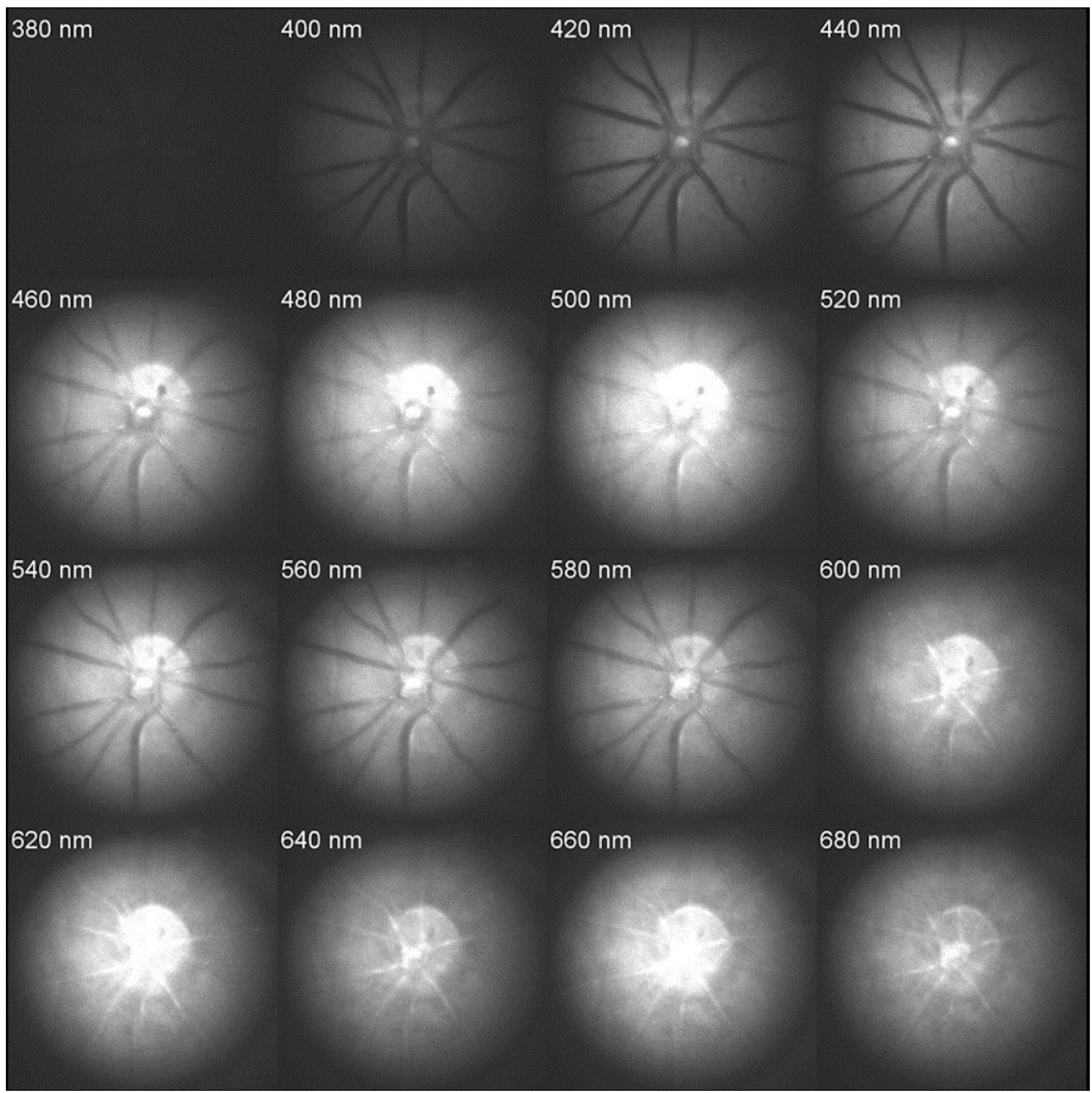

Figure 2. Retinal wavelength series in a representative 6-month-old WT mouse. Monochromatic images were obtained by illuminating the retina using wavelengths $380-680 \mathrm{~nm}$ (only $20 \mathrm{~nm}$ intervals pictured). Notable wavelengths include $480 \mathrm{~nm}$, where the overall image is brightest; $560 \mathrm{~nm}$, where arteries and veins are equally dark; 600-620 nm, where light absorption by oxygenated haemoglobin cause arteries to appear brighter than veins; and $680 \mathrm{~nm}$, where the retinal regions between major blood vessels appear evenly illuminated due to reduced absorption by melanin.

The HSI reflectance for $A \beta$ were similar between those regions of interest including visible aggregates (with speck) and excluding visible aggregates (no speck) (Fig. 1b, Supplementary Table 1, interaction p < 0.001). Moreover, both A $\beta$ regions of interest showed significantly greater HSI reflectance at than vehicle at shorter wavelengths. The largest and most significant difference between A $\beta$ ROIs and vehicle ROIs occurred between 435 to $480 \mathrm{~nm}$ (Fig. 1c, Supplementary Table 1, $\mathrm{p}=0.0021$ to $\mathrm{p}=0.0054$ ). Spectral reflectance at wavelengths from 415 to $505 \mathrm{~nm}$ were significantly different from control (post hoc, $\mathrm{p}<0.05$, Supplementary Table 1 ).

Spectral profile in an in vivo model, 5xFAD mice. Unlike the in vitro preparation, we were unable to visualise discrete aggregates or plaques in the $5 \mathrm{xFAD}$ mouse retinae using HSI (Figs. 2, 3). Other studies using other $A \beta$ animal models have employed contrast agents to identify amyloid plaques, ${ }^{7,46}$. Whether the lack of discrete plaque visualisation was due to the distribution or concentration of $\mathrm{Ab}$ oligomers and plaques in these mice $^{22}$ or the imaging approach employed is unclear. Nevertheless, to achieve an overall gauge of whether the retinal hyperspectral profile was altered in $5 \mathrm{xFAD}$ mice, we analysed whole retinal images to generate spatial maps of relative reflectance differences at short wavelengths (Eq. 3).

As seen from the wavelength series (Fig. 2), the optic nerve and the larger blood vessels in the retina exhibit distinct hyperspectral signatures. There is a wealth of literature showing that the spectral characteristics of the large blood vessels ${ }^{47,48}$ and optic nerve are affected by changes in blood volume, oxygen saturation ${ }^{49,50}$ connective 

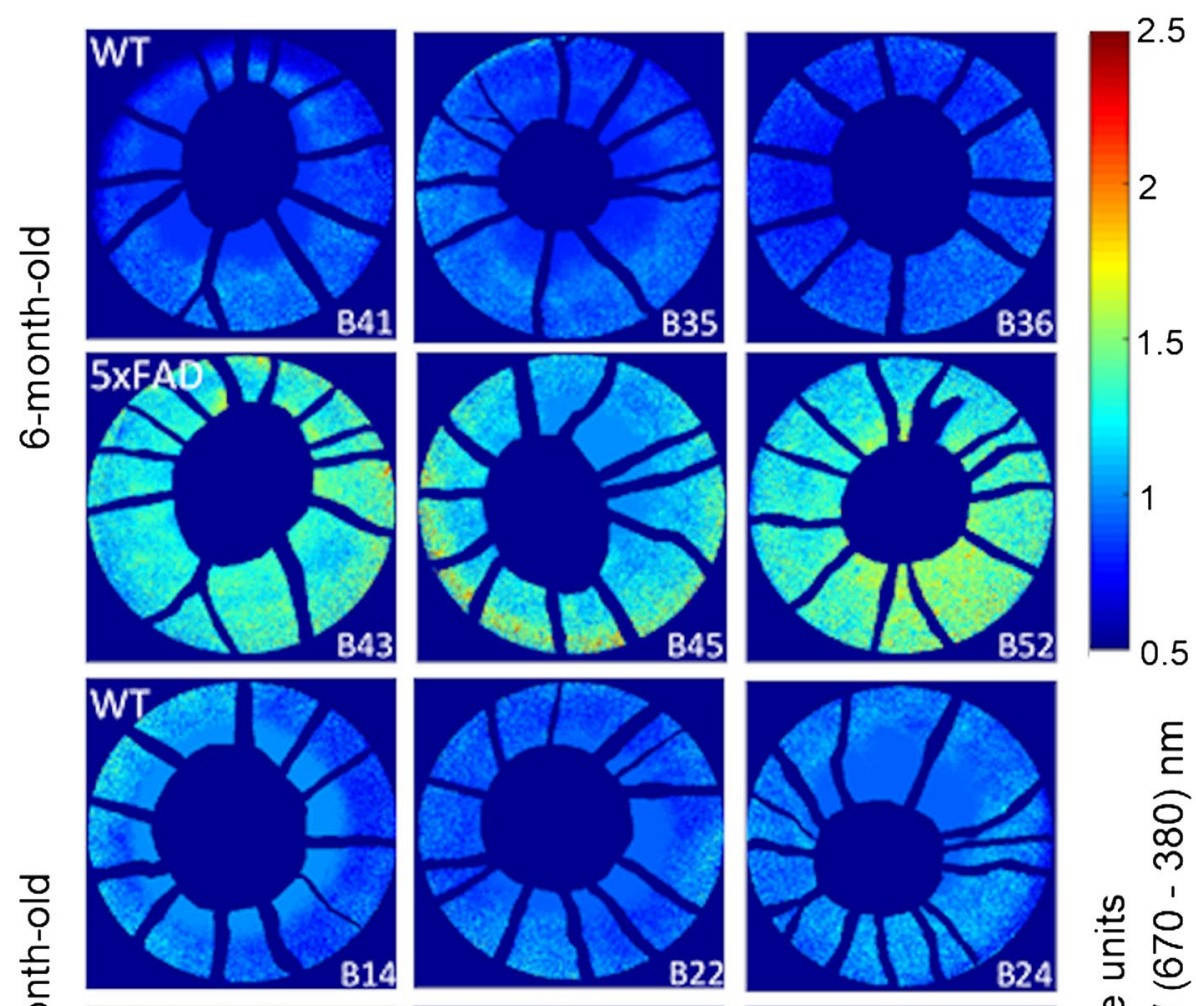

$\frac{E}{\stackrel{\complement}{c}}$

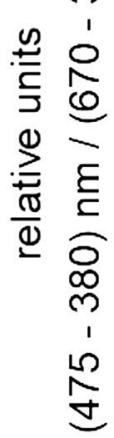
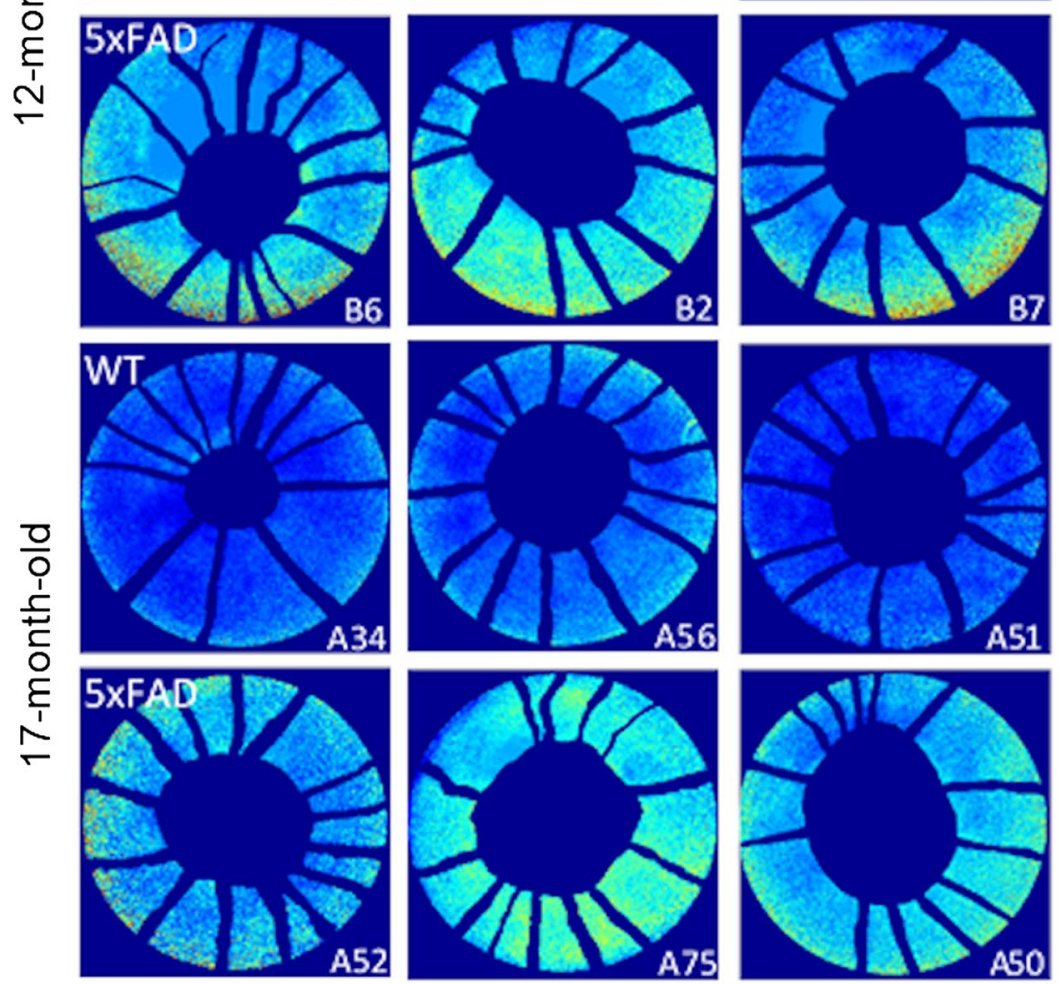

Figure 3. En face retinal images colour coded to reflect areas of greatest spectral difference at $475 \mathrm{~nm}$. Difference plots were generated by expressing the image acquired at $475 \mathrm{~nm}$ relative to the one acquired at $670 \mathrm{~nm}$. Wild type littermates in general showed a ratio closer to 1 (cooler colours), whereas 5xFAD retinae showed ratios of around 1.5 (warmer colours). This difference allows 5xFAD retinae to be visually discernible from WT retinae. 


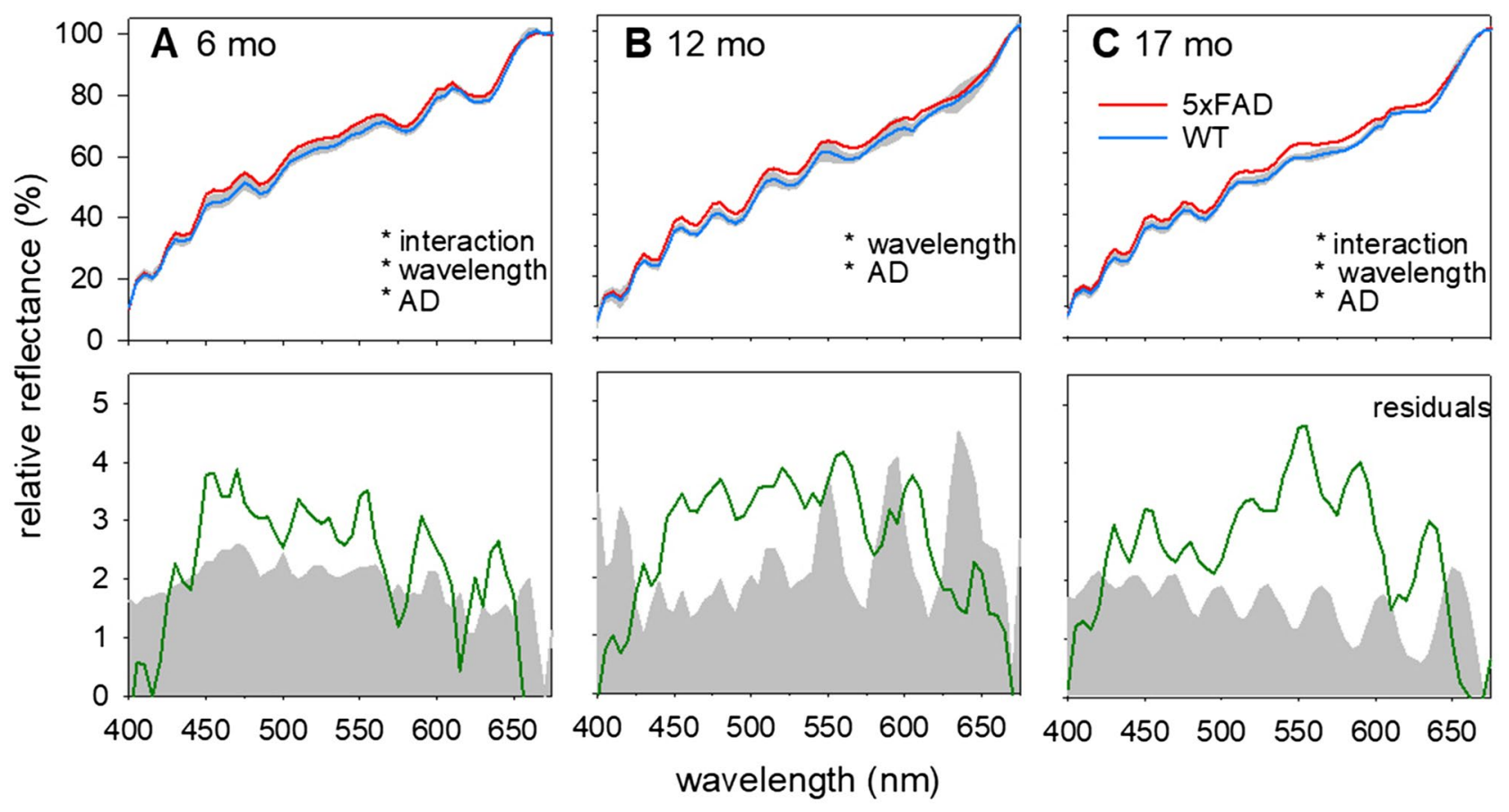

Figure 4. Hyperspectral profile of $5 x F A D$ shows increased relative reflectance compared to WT. In vivo imaging of 5xFAD and WT retinae using hyperspectral imaging $(n=8-15 /$ group $)$ shows a separation between $5 \mathrm{xFAD}$ (red trace) and WT (blue trace) at $6(\mathbf{a}), 12(\mathbf{b})$ and 17 (c) months of age. This difference is plotted as residuals (green line). ${ }^{\star}$ Significant difference using two-way ANOVA; Grey shaded regions, $95 \%$ confidence limits of WT only to aid visualisation.

major blood vessels and the optic nerve area were masked prior to further analysis (Fig. 3).

After the mask was applied, reflectance at $475 \mathrm{~nm}$ was expressed as a ratio of that at $670 \mathrm{~nm}$ for each image pixel (Fig. 3). $475 \mathrm{~nm}$ was chosen as the criterion wavelength as it was representative of increased reflectance when referenced to $670 \mathrm{~nm}$ in both in vitro (Fig. 1) and in vivo preparations (Fig. 4 shows the full profile). This wavelength was similar to More et al. ${ }^{28}$ who used a criterion of $480 \mathrm{~nm}$, with $670 \mathrm{~nm}$ as the reference wavelength. Figure 3 shows that across all ages 5xFAD mice generally exhibit higher relative reflectance at $475 \mathrm{~nm}$ (warmer colours) compared to WT mice. The increased reflectance did not appear to favour specific retinal locations. As our imaging system has a field of view limited to 20 degrees, we were unable to determine if there were greater spectral differences in the peripheral retina compared with the central retina.

Given the lack of region-specific difference, the relative reflectance of the whole retinal image (without blood vessels and the optic nerve) was averaged. Figure 4 compares the hyperspectral profiles between $5 x$ FAD and WT at the three ages. The difference between 5xFAD and WT are plotted as residuals in the lower panels. At all 3 ages, there was increased reflectance in 5xFAD compared with WT across the visible spectrum. At 6 months of age, there was a significant interaction effect between wavelength and genotype (Supplementary Table $2 \mathrm{~A}, \mathrm{p}<0.0001$ ) with a peak difference found at $470 \mathrm{~nm}$ and $5 \mathrm{xFAD}$ mice deviated from WT fairly consistently between 450-490 nm (Fig. 4A, Supplementary Table 2A). At 12 months, increased reflectance toward shorter wavelengths $(<490 \mathrm{~nm})$ and a second hump around $\sim 550 \mathrm{~nm}$ was also observed (Fig. 4b, Supplementary Table 2). Using two-way ANOVA, the spectral differences were explained by wavelength and genotype (wavelength effect, $\mathrm{p}<0.0001$, genotype effect, $\mathrm{p}<0.05)$. At 17 months of age, the greatest differences were observed at longer wavelengths $\sim 555 \mathrm{~nm}$ spanning a $510-560 \mathrm{~nm}$ band and a smaller peak at shorter wavelengths (Fig. $4 \mathrm{c}$, Supplementary Table 2C).

The main differences in retinal reflectance between 6-12 5xFAD and WT mice appeared around shorter wavelengths $(<500 \mathrm{~nm})$ as well as a second peak and longer wavelengths $\sim 550-560 \mathrm{~nm}$. The increased reflectance at short wavelengths is consistent with the in vitro data seen in Fig. 1 . The reflectance difference at short wavelengths was less apparent at 17 months of age, and at this age the difference at longer wavelengths $(>550 \mathrm{~nm})$ was more apparent. Difference between 5xFAD mice of different ages are most obvious when the residuals (A $\beta$ - Control) are plotted along the same axis (Fig. 5). In order to more easily visualize the ageing shift towards increased reflectance at longer wavelengths, Fig. 5 shows the residuals normalized to an approximate band around the short wavelength peaks of different ages and preparations (average residual between $450-475 \mathrm{~nm}$ ). This approach also facilitates comparison of in vivo and in vitro $A \beta$ reflectivity.

\section{Discussion}

Change in hyperspectral reflectivity at short wavelengths. This study shows increased relative reflectance at shorter wavelengths $(<500 \mathrm{~nm})$ may be a signature of retinal changes associated with different $A \beta$ preparations. This was relatively consistent when we compared hyperspectral imaging of isolated human $A \beta 42$ 


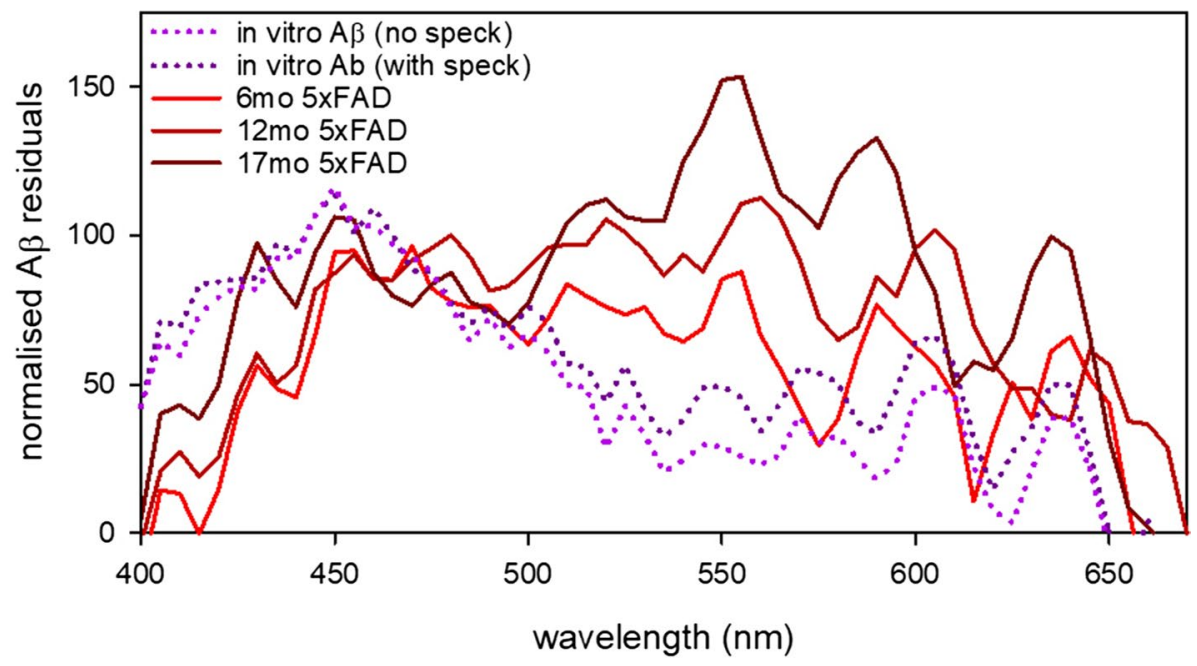

Figure 5. Normalised residual plots showcasing difference between control and amyloid in in vitro and in vivo conditions. Data normalised to a band between 450 and $475 \mathrm{~nm}$ to visualise profiles between different preparations.

(in vitro) in a drop of water to in vivo retinal imaging in a transgenic murine model shown to exhibit $A \beta$ in the retina. That the presence of $A \beta$ increases reflectance at shorter wavelengths is consistent with a recent clinical study reporting that participants with mild cognitive impairment and high cortical A $\beta$ load (PET imaging) exhibited increased reflectance at short wavelengths compared to age matched controls ${ }^{30}$. That study modelled optical media, posterior pole pigmentation, and haemoglobin (using Dimension Reduction by Orthogonal Projection for Discrimination, DROP-D) all of which modify retinal reflectance between individuals. The need for such modelling may be less important in rodent eyes given the greater homogeneity within an animal model and the absence of macular pigmentation. It is of note that increased reflectance in 5xFAD mice was less apparent at 17 months of age than at 6 and 12 months (Fig. 4), which may be due to absorbance of shorter wavelengths by the crystalline lens ${ }^{51}$.

More and colleagues reported a decrease in reflectance of short wavelengths $\sim 480$ to $550 \mathrm{~nm}$, in a human neuroblastoma cell line ${ }^{27}$ and in APP/PS1 AD mice ${ }^{28}$. This is an effect opposite to that observed in the current study and that of Hadoux et al. ${ }^{30}$ The current study and Hadoux et al ${ }^{30}$ employed perpendicular bright field illumination whereas More and Vince ${ }^{27}$ used a system analogous to a darkfield microscope. This different lighting and imaging geometry might explain why their system detected reduced reflectance at the short wavelengths with increasing A $\beta 42$ aggregation. In contrast, our and Hadoux et al. ${ }^{30}$ approach employing a simple ophthalmoscope, resulted in increased reflectance at short wavelengths.

Using a LED based interferometric system with broadband incident light at $0^{\circ}$, Cheng et al. ${ }^{52}$ showed a shift towards increased total reflectance associated with increased soluble $A \beta$. Reflectance was further increased by the addition of fibril promoting metal compounds (e.g. zinc), whereas reduced reflectance was seen in the presence of polyphenols (e.g. (-)-epigallocatechin-3-gallate), which prevents fibril elongation. In another study, Yu et al. ${ }^{53}$, using a spectrofluorometer with a light source placed directly behind the sample $\left(0^{\circ}\right.$ incident beam $)$ showed that particles (gold-coated iron oxide) with diameters of $320 \mathrm{~nm}$ in the UV-VIS range when coated with increasing concentrations of $A \beta$ resulted in increasing reflectance.

Hyperspectral imaging with advancing age. Figures 4 and 5 illustrate how the shape of the HSI profile modifies with age. All profiles show an increase in reflectance at shorter wavelengths. By normalising the residuals to a band between 450 and 475 it can be seen that with advancing age the 5xFAD mice show a relatively greater reflection at longer wavelengths as well. A previous study by our group quantified immunohistochemical staining of $A \beta$ plaques and oligomers in a sub-set of these $5 x F A D$ mice ${ }^{22}$. This study showed that oligomer levels were highest at 6 months of age and then gradually declined at 12 and 14-17 months of age. In contrast $\mathrm{A} \beta$ plaque deposition gradually increased with advancing age. This pattern was mirrored in hippocampal and cortical tissues. Age-matched control tissue exhibited lower levels of A $\beta$ staining than tissue from 5xFAD counterparts. These findings support the idea that younger mice exhibit increased reflectance at shorter wavelengths corresponding to oligomer induced light scatter (Figs. 4a, 5). With advancing age, the increased reflectance (Figs. 4b,c, 5) at short wavelengths is less prominent and reflectance at longer wavelengths (>550 $\mathrm{nm})$ increases, which may be associated with $A \beta$ plaque induced scatter.

This study also examines the spectral profile of human A $\beta 42$ when suspended in PBS. We show that amyloid is consistent with a light scattering effect, resulting in increased light reflectance at shorter wavelengths of the visible light spectrum (Fig. 1). When comparing those ROIs in the in vitro preparation that included visible aggregates and background solution versus those ROIs where only the background solution was visible produced similar profiles. There was a trend for those ROIs which included visible specks to have increased reflectivity 
at longer wavelengths consistent with the $5 \mathrm{xFAD}$ data's progression with advancing age however this trend was minimal and not significant.

\section{Limitations}

When considering in vivo spectroscopy, it is also possible that other biological factors come into play. The 5xFAD data were normalised to a long wavelength based on previous literature of $A \beta$-aggregates in cell culture ${ }^{27}$, excised brain and retinal tissue ${ }^{27}$, in-vivo mouse tissue ${ }^{28}$, and our in vitro data (Fig. 1). However, for completeness and to additionally consider other non-A $\beta$ influences on the HSI profiles the data was also normalized to the average reflectance of all the wavelengths (Supplementary Fig. 3). In this manner, variability from differences in overall reflectivity can be removed whilst making no assumptions as to what is causing reflectivity changes or at what wavelengths they occur. This alternate approach (Supplementary Fig. 3) shows a similar shaped profile to Fig. 4, albeit shifted downwards. Regardless of the normalisation approach, the observation that at shorter wavelengths the $5 \mathrm{xFAD}$ mice show increased reflectivity; and with advancing age there is an additional peak at $\sim 550 \mathrm{~nm}$ remains consistent. However, the approach of normalising to the average reflectivity shows a reduction in reflectivity at long wavelengths $(>600 \mathrm{~nm})$ not seen when normalised to $670 \mathrm{~nm}$.

Altered oxygenation causes changes to retinal reflectivity in this wavelength range. $600-750 \mathrm{~nm}^{54}$. Although major blood vessels were masked, retinal capillaries spread across the retina making altered tissue oxygenation a possible driver of changes seen at wavelengths above $600 \mathrm{~nm}$. In a subset of animals, partial pressure of oxygen (PO2) was measured and no significant difference were noted at any age between 5xFAD mice and WT littermates (Supplementary Table 3), making this explanation perhaps less likely. Note that pO2 analysis was conducted under general anesthesia analogous to that used during hyperspectral imaging. Nevertheless, to conclusively determine whether altered oxygenation is driving the hyperspectral changes at long wavelengths or whether it is a mathematical manifestation of normalising to the centre of the spectrum as opposed to the end requires further interventional studies such as hyperoxic breathing.

Another important factor to consider with retinal imaging is the effect of age-related cataract formation in older eyes, which can mask light scatter at shorter wavelengths. Although age-matched control animals were used as a comparison it is possible that $5 \mathrm{xFAD}$ mice may exhibit greater anterior eye opacities and thus contribute to the difference in the 17 -month-old age group compared with younger $5 x F A D$ mice. Additionally, the retinal nerve fibre layer (RNFL) and in particular the microtubules within ganglion cell axons reflect a significant amount of light in the short (415-440 nm) visible wavelength spectrum ${ }^{55-58}$. In humans with AD and animal models of the disease, RNFL thinning has been reported ${ }^{59-62}$. However, RNFL thinning should reduce reflectance between 415 and $440 \mathrm{~nm}$, which is inconsistent with the increased reflectance observed in this study. Nevertheless, to determine the exact contributions of increased short and mid wavelength reflectivity, future interventional studies are required.

\section{Conclusions}

We describe in this study a simple and non-invasive biomarker for the detection of amyloid-related changes in a murine model of AD. This was achieved without the administration of a contrast agent and did not require complex mathematical modelling. Our observations in younger 5xFAD mice suggest that increased reflectance towards shorter wavelengths coincides with when oligomer levels are highest in the retina. With advancing age, the reflectance at longer wavelengths increases when $A \beta$ plaques show greater deposition in the retina. Future studies are required to determine whether this observation is causal in nature, and whether it may be a useful metric for staging Alzheimer's disease.

\section{Data availability}

The authors confirm that we will adhere to the journals data availability policies including making materials, data and associated protocols promptly available to readers without undue qualifications in material transfer agreements. The authors also disclose a subset of the animals used in this manuscript had separate techniques conducted on them (electroretinography, optical coherence tomography) which was published in another manuscript (Lim et al. 2020, Frontier in Neuroscience) as well as their tissue taken and analysed (Habiba et al. 2020, Journal of Alzheimer's Disease).

Received: 19 June 2020; Accepted: 20 February 2021

Published online: 18 March 2021

\section{References}

1. Nakamura, A. et al. High performance plasma amyloid-beta biomarkers for Alzheimer's disease. Nature 554, 249-254. https://doi. org/10.1038/nature25456 (2018).

2. Schindler, S. E. et al. High-precision plasma beta-amyloid $42 / 40$ predicts current and future brain amyloidosis. Neurology $\mathbf{9 3}$, e1647-e1659. https://doi.org/10.1212/WNL.0000000000008081 (2019).

3. Lim, J. K. et al. The Eye As a Biomarker for Alzheimer's Disease. Front Neurosci 10, 536. https://doi.org/10.3389/fnins.2016.00536 (2016).

4. Alber, J. et al. Developing retinal biomarkers for the earliest stages of Alzheimer's disease: What we know, what we don't, and how to move forward. Alzheimer's \& dementia : the journal of the Alzheimer's Association 16, 229-243. https://doi.org/10.1002/alz.12006 (2020).

5. Loffler, K. U., Edward, D. P. \& Tso, M. O. Immunoreactivity against tau, amyloid precursor protein, and beta-amyloid in the human retina. Invest. Ophthalmol. Vis. Sci. 36, 24-31 (1995).

6. Koronyo, Y. et al. Retinal amyloid pathology and proof-of-concept imaging trial in Alzheimer's disease. JCI Insight 2, https://doi. org/10.1172/jci.insight.93621 (2017). 
7. Koronyo-Hamaoui, M. et al. Identification of amyloid plaques in retinas from Alzheimer's patients and noninvasive in vivo optical imaging of retinal plaques in a mouse model. Neuroimage 54(Suppl 1), S204-217. https://doi.org/10.1016/j.neuroimage.2010.06. $020(2011)$

8. La Morgia, C. et al. Melanopsin retinal ganglion cell loss in Alzheimer disease. Ann. Neurol. 79, 90-109. https://doi.org/10.1002/ ana.24548 (2016).

9. Ho, C. Y., Troncoso, J. C., Knox, D., Stark, W. \& Eberhart, C. G. Beta-Amyloid, Phospho-Tau and Alpha-Synuclein Deposits Similar to Those in the Brain Are Not Identified in the Eyes of Alzheimer's and Parkinson's Disease Patients. Brain Pathol https://doi.org/ $10.1111 /$ bpa.12070 (2013).

10. Schon, C. et al. Long-term in vivo imaging of fibrillar tau in the retina of P301S transgenic mice. PLoS ONE 7, e53547. https://doi. org/10.1371/journal.pone.0053547 (2012).

11. Williams, E. A. et al. Absence of Alzheimer Disease Neuropathologic Changes in Eyes of Subjects With Alzheimer Disease. J Neuropathol Exp Neurol 76, 376-383. https://doi.org/10.1093/jnen/nlx020 (2017).

12. Gupta, V. K. et al. Amyloid beta accumulation and inner retinal degenerative changes in Alzheimer's disease transgenic mouse. Neurosci. Lett. 623, 52-56. https://doi.org/10.1016/j.neulet.2016.04.059 (2016).

13. Parthasarathy, R. et al. Reduction of amyloid-beta levels in mouse eye tissues by intra-vitreally delivered neprilysin. Exp Eye Res 138, 134-144. https://doi.org/10.1016/j.exer.2015.06.027 (2015).

14. Alexandrov, P. N., Pogue, A., Bhattacharjee, S. \& Lukiw, W. J. Retinal amyloid peptides and complement factor H in transgenic models of Alzheimer's disease. NeuroReport 22, 623-627. https://doi.org/10.1097/WNR.0b013e3283497334 (2011).

15. Dutescu, R. M. et al. Amyloid precursor protein processing and retinal pathology in mouse models of Alzheimer's disease. Graefe's archive for clinical and experimental ophthalmology = Albrecht von Graefes Archiv fur klinische und experimentelle Ophthalmologie 247, 1213-1221. https://doi.org/10.1007/s00417-009-1060-3 (2009).

16. Hoh Kam, J., Lenassi, E. \& Jeffery, G. Viewing ageing eyes: diverse sites of amyloid Beta accumulation in the ageing mouse retina and the up-regulation of macrophages. PLoS One 5. https://doi.org/10.1371/journal.pone.0013127 (2010).

17. Perez, S. E., Lumayag, S., Kovacs, B., Mufson, E. J. \& Xu, S. Beta-amyloid deposition and functional impairment in the retina of the APPswe/PS1DeltaE9 transgenic mouse model of Alzheimer's disease. Invest. Ophthalmol. Vis. Sci. 50, 793-800. https://doi. org/10.1167/iovs.08-2384 (2009).

18. Ning, A., Cui, J., To, E., Ashe, K. H. \& Matsubara, J. Amyloid-beta deposits lead to retinal degeneration in a mouse model of Alzheimer disease. Invest. Ophthalmol. Vis. Sci. 49, 5136-5143. https://doi.org/10.1167/iovs.08-1849 (2008).

19. Tsai, Y. et al. Ocular changes in TgF344-AD rat model of Alzheimer's disease. Invest. Ophthalmol. Vis. Sci. 55, 523-534. https:// doi.org/10.1167/iovs.13-12888 (2014).

20. Du, L. Y. et al. Alzheimer's Disease-Related Protein Expression in the Retina of Octodon degus. PLoS ONE 10, e0135499. https:// doi.org/10.1371/journal.pone.0135499 (2015).

21. Grimaldi, A. et al. Inflammation, neurodegeneration and protein aggregation in the retina as ocular biomarkers for Alzheimer's disease in the 3xTg-AD mouse model. Cell Death Dis 9, 685. https://doi.org/10.1038/s41419-018-0740-5 (2018).

22. Habiba, U. et al. Age-Specific Retinal and Cerebral Immunodetection of Beta Amyloid Plaques and Oligomers in a Rodent Model of Alzheimer's Disease. Journal of Alzheimer's Disease Accepted 04/06/2020 (2020).

23. Campbell, M. et al. in Bio-Optics: Design and Application BM3A. 4 (Optical Society of America, Vancouver, Canada, 2015).

24. Campbell, M. et al. Distribution of Amyloid Deposits across the Retina in Association with Alzheimer's Disease as a Function of Disease Severity. Alzheimers Dement. 15, P685-P687. https://doi.org/10.1016/j.jalz.2019.06.2669 (2019).

25. Campbell, M. et al. Amyloid Deposits Imaged in Postmortem Retinas Using Polarimetry Predict the Severity of a Postmortem Brain Based Diagnosis of Alzheimer's Disease. Alzheimers Dement. 14, P774-P775. https://doi.org/10.1016/j.jalz.2018.06.947 (2018).

26. Jacques, S. L. Optical properties of biological tissues: a review. Phys Med Biol 58, R37-61. https://doi.org/10.1088/0031-9155/58/ $11 / \mathrm{r} 37$ (2013).

27. More, S. S. \& Vince, R. Hyperspectral imaging signatures detect amyloidopathy in Alzheimer's mouse retina well before onset of cognitive decline. ACS Chem. Neurosci. 6, 306-315. https://doi.org/10.1021/cn500242z (2015).

28. More, S. S., Beach, J. M. \& Vince, R. Early Detection of Amyloidopathy in Alzheimer's Mice by Hyperspectral Endoscopy. Invest Ophthalmol Vis Sci 57, 3231-3238. https://doi.org/10.1167/iovs.15-17406 (2016).

29. Shimazawa, M. et al. Reduced retinal function in amyloid precursor protein-over-expressing transgenic mice via attenuating glutamate-N-methyl-d-aspartate receptor signaling. J. Neurochem. 107, 279-290. https://doi.org/10.1111/j.1471-4159.2008.05606.x (2008).

30. Hadoux, X. et al. Non-invasive in vivo hyperspectral imaging of the retina for potential biomarker use in Alzheimer's disease. Nat Commun 10, 4227. https://doi.org/10.1038/s41467-019-12242-1 (2019).

31. Oakley, H. et al. Intraneuronal beta-amyloid aggregates, neurodegeneration, and neuron loss in transgenic mice with five familial Alzheimer's disease mutations: potential factors in amyloid plaque formation. The Journal of neuroscience : the official journal of the Society for Neuroscience 26, 10129-10140. https://doi.org/10.1523/jneurosci.1202-06.2006 (2006).

32. O'Leary, T. P., Mantolino, H. M., Stover, K. R. \& Brown, R. E. Age-related deterioration of motor function in male and female 5xFAD mice from 3 to 16 months of age. Genes Brain Behav, e12538. https://doi.org/10.1111/gbb.12538 (2018).

33. Flanigan, T. J., Xue, Y., Kishan Rao, S., Dhanushkodi, A. \& McDonald, M. P. Abnormal vibrissa-related behavior and loss of barrel field inhibitory neurons in 5xFAD transgenics. Genes Brain Behav 13, 488-500. https://doi.org/10.1111/gbb.12133 (2014).

34. Kimura, R. \& Ohno, M. Impairments in remote memory stabilization precede hippocampal synaptic and cognitive failures in 5XFAD Alzheimer mouse model. Neurobiol Dis 33, 229-235. https://doi.org/10.1016/j.nbd.2008.10.006 (2009).

35. Percie du Sert, N. et al. The ARRIVE guidelines 2.0: Updated guidelines for reporting animal research. PLoS Biol 18, e3000410. https://doi.org/10.1371/journal.pbio.3000410 (2020).

36. McColl, G. et al. The Caenorhabditis elegans A $\beta(1-42)$ Model of Alzheimer Disease Predominantly Expresses A $\beta(3-42)$. J. Biol. Chem. 284, 22697-22702. https://doi.org/10.1074/jbc.C109.028514 (2009).

37. Ryan, T. M. et al. Ammonium hydroxide treatment of Abeta produces an aggregate free solution suitable for biophysical and cell culture characterization. PeerJ 1, e73. https://doi.org/10.7717/peerj.73 (2013).

38. Ryan, T. M. et al. Small amphipathic molecules modulate secondary structure and amyloid fibril-forming kinetics of Alzheimer disease peptide Abeta(1-42). J Biol Chem 287, 16947-16954. https://doi.org/10.1074/jbc.M111.321778 (2012).

39. Park, S. W. et al. Dry age-related macular degeneration like pathology in aged 5XFAD mice: Ultrastructure and microarray analysis. Oncotarget 8, 40006-40018. https://doi.org/10.18632/oncotarget.16967 (2017).

40. Penn, J. S., Naash, M. I. \& Anderson, R. E. Effect of light history on retinal antioxidants and light damage susceptibility in the rat. Exp. Eye Res. 44, 779-788. https://doi.org/10.1016/s0014-4835(87)80041-6 (1987).

41. Ishida, A. et al. Light activates the adrenal gland: timing of gene expression and glucocorticoid release. Cell Metab 2, 297-307. https://doi.org/10.1016/j.cmet.2005.09.009 (2005).

42. Focus Technologies, L. Maximum permissable exposure., (2013).

43. Schindelin, J. et al. Fiji: an open-source platform for biological-image analysis. Nat Methods 9, 676-682. https://doi.org/10.1038/ nmeth.2019 (2012).

44. Benjamini, Y. \& Hochberg, Y. Controlling the false discovery rate: a practical and powerful approach to multiple testing. Journal of the Royal Statistical Society. Series B (Methodological), 289-300 (1995). 
45. Benjamini, Y., Krieger, A. \& Yekutieli, D. Adaptive linear step-up procedures that control the false discovery rate. Biometrika 93, 491-507 (2006).

46. Emptage, L. et al. in ARVO 2016 Annual Meeting Vol. Abstract \#2218 (Seattle, 2016)

47. Beach, J. M., Schwenzer, K. J., Srinivas, S., Kim, D. \& Tiedeman, J. S. Oximetry of retinal vessels by dual-wavelength imaging: calibration and influence of pigmentation. J. Appl. Physiol. 86, 748-758 (1999).

48. Chen, S., Yi, J., Liu, W., Backman, V. \& Zhang, H. F. Monte Carlo Investigation of Optical Coherence Tomography Retinal Oximetry. IEEE Trans Biomed Eng 62, 2308-2315. https://doi.org/10.1109/tbme.2015.2424689 (2015).

49. Denniss, J. et al. Relationships between Visual Field Sensitivity and Spectral Absorption Properties of the Neuroretinal Rim in Glaucoma by Multispectral Imaging. Invest. Ophthalmol. Vis. Sci. 52, 8732-8738. https://doi.org/10.1167/iovs.11-8302 (2011).

50. Bambo, M. P. et al. Analysis of optic disk color changes in Alzheimer's disease: a potential new biomarker. Clin Neurol Neurosurg 132, 68-73. https://doi.org/10.1016/j.clineuro.2015.02.016 (2015).

51. Douglas, R. H. \& Jeffery, G. The spectral transmission of ocular media suggests ultraviolet sensitivity is widespread among mammals. Proc Biol Sci 281, 20132995. https://doi.org/10.1098/rspb.2013.2995 (2014).

52. Cheng, X. R., Daaboul, G. G., Unlu, M. S. \& Kerman, K. LED-based interferometric reflectance imaging sensor for the detection of amyloid-beta aggregation. Analyst 139, 59-65. https://doi.org/10.1039/c3an01307c (2014).

53. Yu, L. et al. A highly sensitive resonance light scattering probe for Alzheimer's amyloid-beta peptide based on Fe3O4@Au composites. Talanta 131, 475-479. https://doi.org/10.1016/j.talanta.2014.07.067 (2015).

54. Nitzan, M. et al. Calibration-free pulse oximetry based on two wavelengths in the infrared - a preliminary study. Sensors (Basel) 14, 7420-7434. https://doi.org/10.3390/s140407420 (2014).

55. Zhou, Q. \& Knighton, R. W. Light scattering and form birefringence of parallel cylindrical arrays that represent cellular organelles of the retinal nerve fiber layer. Appl Opt 36, 2273-2285 (1997).

56. Huang, X. R., Knighton, R. W. \& Cavuoto, L. N. Microtubule contribution to the reflectance of the retinal nerve fiber layer. Invest Ophthalmol Vis Sci 47, 5363-5367. https://doi.org/10.1167/iovs.06-0451 (2006).

57. Knighton, R. W., Huang, X. \& Zhou, Q. Microtubule contribution to the reflectance of the retinal nerve fiber layer. Invest. Ophthalmol. Vis. Sci. 39, 189-193 (1998).

58. Zhang, X. et al. Dual-band spectral-domain optical coherence tomography for in vivo imaging the spectral contrasts of the retinal nerve fiber layer. Opt Express 19, 19653-19659. https://doi.org/10.1364/OE.19.019653 (2011).

59. den Haan, J., Verbraak, F. D., Visser, P. J. \& Bouwman, F. H. Retinal thickness in Alzheimer's disease: A systematic review and meta-analysis. Alzheimers Dement (Amst) 6, 162-170. https://doi.org/10.1016/j.dadm.2016.12.014 (2017).

60. Almeida, A. L. M. et al. Correlation between cognitive impairment and retinal neural loss assessed by swept-source optical coherence tomography in patients with mild cognitive impairment. Alzheimers Dement (Amst) 11, 659-669. https://doi.org/10.1016/j. dadm.2019.08.006 (2019).

61. Lopez-de-Eguileta, A. et al. Ganglion cell layer thinning in prodromal Alzheimer's disease defined by amyloid PET. Alzheimers Dement (N Y) 5, 570-578. https://doi.org/10.1016/j.trci.2019.08.008 (2019).

62. Chiquita, S. et al. Retinal thinning of inner sub-layers is associated with cortical atrophy in a mouse model of Alzheimer's disease: a longitudinal multimodal in vivo study. Alzheimer's research \& therapy 11, 90. https://doi.org/10.1186/s13195-019-0542-8 (2019).

\section{Acknowledgements}

The authors acknowledge funding through an Australian Research Council Linkage grant LP160100126 and funding partners AstraZeneca (Craig Shering, Jamie Mullen, Gvido Cebers) and Biogen Inc (Mike Wald, Nicolas Currier, Samantha Budd Haeberlein, Richard Hargreaves). Funding partners AstraZeneca and Biogen Inc. were additionally involved in the conceptualization of the work program leading to this publication.

\section{Author contributions}

J.L., B.B., C.N. contributed to the conceptualisation of the study, data collection, data analysis and writing of the manuscript. Q.L., T.R. contributed to data collection and writing of the manuscript. P.B., A.M., A.V. contributed to the data analysis and writing of the manuscript.

\section{Funding}

C.N., B.B., A.V.: ARC Linkage grant LP160100126, C.N.: Melbourne Research Fellowship, Melbourne Neuroscience Institute Fellowship, Melbourne School of Health Sciences Seed funding JL: Guelma-Alexander Clinical Fellowship in Neuroscience 2019, Melbourne Neuroscience Institute Strategic Postgraduate Award (STRAPA).

\section{Competing interests}

$\mathrm{CN}, \mathrm{BB}, \mathrm{AV}, \mathrm{GC}$ (acknowledgement), SH (acknowledgement) are joint investigators on an Australian Research Council Linkage grant LP160100126 with funding partners AstraZeneca and Biogen Inc. MW, NC, SH, RH (all acknowledgement) are employees of Biogen Inc. CS, JM, GC (all acknowledgement) are employees or former employees of AstraZeneca.

\section{Additional information}

Supplementary Information The online version contains supplementary material available at https://doi.org/ 10.1038/s41598-021-85554-2.

Correspondence and requests for materials should be addressed to C.T.O.N.

Reprints and permissions information is available at www.nature.com/reprints.

Publisher's note Springer Nature remains neutral with regard to jurisdictional claims in published maps and institutional affiliations. 
(c) (i) Open Access This article is licensed under a Creative Commons Attribution 4.0 International cc) License, which permits use, sharing, adaptation, distribution and reproduction in any medium or format, as long as you give appropriate credit to the original author(s) and the source, provide a link to the Creative Commons licence, and indicate if changes were made. The images or other third party material in this article are included in the article's Creative Commons licence, unless indicated otherwise in a credit line to the material. If material is not included in the article's Creative Commons licence and your intended use is not permitted by statutory regulation or exceeds the permitted use, you will need to obtain permission directly from the copyright holder. To view a copy of this licence, visit http://creativecommons.org/licenses/by/4.0/.

(C) The Author(s) 2021 